\title{
Gas and dust mass in the disc around the Herbig Ae star HD 169142
}

\author{
O. Panić ${ }^{1}$, M. R. Hogerheijde ${ }^{1}$, D. Wilner ${ }^{2}$, and C. Qi $^{2}$ \\ 1 Leiden Observatory, Leiden University, PO Box 9513, 2300 RA, Leiden, The Netherlands \\ e-mail: olja@strw.leidenuniv.nl \\ 2 Harvard-Smithsonian Center for Astrophysics, 60 Garden Street, Cambridge, MA 02138, USA
}

Received 17 December 2007 / Accepted 22 May 2008

\begin{abstract}
Context. Spatially resolved observations of circumstellar discs at millimetre wavelengths allow detailed comparisons with theoretical models for the radial and vertical distribution of the material.

Aims. We investigate the physical structure of the gas component of the disc around the pre-main-sequence star HD 169142 and test the disc model derived from the spectral energy distribution.

Methods. The ${ }^{13} \mathrm{CO}$ and $\mathrm{C}^{18} \mathrm{O} J=2-1$ line emission was observed from the disc with 1".4 resolution using the Submillimeter Array. We adopted the disc physical structure derived from a model that fits the spectral energy distribution of HD 169142. We obtained the full three-dimensional information on the $\mathrm{CO}$ emission with the aid of a molecular excitation and radiative transfer code. This information was used for the analysis of our observations and previous ${ }^{12} \mathrm{CO} J=2-1$ and $1.3 \mathrm{~mm}$ continuum data.

Results. The spatially resolved ${ }^{13} \mathrm{CO}$ and $\mathrm{C}^{18} \mathrm{O}$ emission shows a Keplerian velocity pattern. The disc is seen at an inclination close to $13^{\circ}$ from face-on. We conclude that the regions traced by different $\mathrm{CO}$ isotopologues are distinct in terms of their vertical location within the disc, their temperature, and their column densities. With the given disc structure, we find that freeze-out is not efficient enough to remove a significant amount of $\mathrm{CO}$ from the gas phase. Both observed lines match the model prediction both in flux and in the spatial structure of the emission. Therefore we use our data to derive the ${ }^{13} \mathrm{CO}$ and $\mathrm{C}^{18} \mathrm{O}$ mass and consequently the ${ }^{12} \mathrm{CO}$ mass with standard isotopic ratios. We constrain the total disc gas mass to $(0.6-3.0) \times 10^{-2} M_{\odot}$. Adopting a maximum dust opacity of $2 \mathrm{~cm}^{2} \mathrm{~g}_{\text {dust }}^{-1}$ we derive a minimum dust mass of $2.16 \times 10^{-4} M_{\odot}$ from the fit to the $1.3 \mathrm{~mm}$ data. Comparison of the derived gas and dust mass shows that the gas-to-dust mass ratio of 100 is only possible under the assumption of a dust opacity of $2 \mathrm{~cm}^{2} \mathrm{~g}^{-1}$ and ${ }^{12} \mathrm{CO}$ abundance of $10^{-4}$ with respect to $\mathrm{H}_{2}$. However, our data are also compatible with a gas-to-dust ratio of 25 , with a dust opacity of $1 \mathrm{~cm}^{2} \mathrm{~g}^{-1}$ and ${ }^{12} \mathrm{CO}$ abundance of $2 \times 10^{-4}$.
\end{abstract}

Key words. astrochemistry - techniques: interferometric - planetary systems: protoplanetary disks - stars: individual: HD $169142-$ stars: pre-main sequence - submillimeter

\section{Introduction}

Although the presence of molecular gas in discs around intermediate mass pre-main-sequence (Herbig Ae) stars was reported a decade ago (Mannings \& Sargent 1997), the research in this field has focused more on their less massive counterparts (T Tauri stars). The discs around T Tauri stars have masses ranging from 0.001 to $0.1 M_{\odot}$ (Beckwith et al. 1990), usually derived from millimetre continuum fluxes assuming a gas-todust mass ratio of 100 , as in molecular clouds. Their outer radii are constrained by molecular line observations and are typically a few hundred AU (Simon et al. 2000; Thi et al. 2001, and references therein). Due to the low luminosity of the central star $\left(0.5\right.$ to $\left.1 L_{\odot}\right)$, these discs are relatively cold (less than $20 \mathrm{~K}$ beyond $100 \mathrm{AU}$ from the star) causing a significant depletion of the $\mathrm{CO}$ in the midplane of the outer disc. On the other hand, the Herbig Ae stars are about ten times more luminous than T Tauri stars, and consequently their discs are warmer. This allows the $\mathrm{CO}$, the easiest to detect and the most commonly used gas tracer, to remain in the gas phase even in the disc midplane. Observations of $\mathrm{CO}$ and its isotopologues toward Herbig Ae stars are therefore expected to be more powerful probes of the full disc structure. Only a few Herbig Ae discs have been studied thoroughly via spatially resolved observations of molecular line emission that includes the optically thin CO isotopologues: AB Aur (Piétu et al. 2005), MWC 480 (Piétu et al. 2007), and HD 163296 (Isella et al. 2007).

\section{HD 169142}

The object of our study, HD 169142, is a $2.0 M_{\odot}$ Herbig Ae star of spectral type A5Ve surrounded by a gas-rich circumstellar disc located at $145 \mathrm{pc}$ (Sylvester et al. 1996). With an age of $6_{-3}^{+6}$ Myr (Grady et al. 2007) and its spectral energy distribution marked by infrared excess and the lack of silicate features (Dent et al. 2006), HD 169142 is an example of an advanced pre-mainsequence evolutionary stage. Unlike most of the Herbig Ae/Be stars, it shows no evidence of proximity to a cloud or extended molecular gas (Meeus et al. 2001). Observations of molecular gas in this disc are therefore easier to interpret. However, HD 169142 is not completely isolated from other young stars; e.g., Grady et al. (2007) find three coeval pre-main-sequence stars within a projected separation of $1160 \mathrm{AU}$. The closest companion is located at 9!3 separation and may form a binary system with HD 169142. Near-infrared polarisation images show that the dust in the disc extends to at least 217 AU (Kuhn et al. 2001). More recent submillimetre observations (Dent et al. 2005; Raman et al. 2006) show bright and narrow CO lines. 


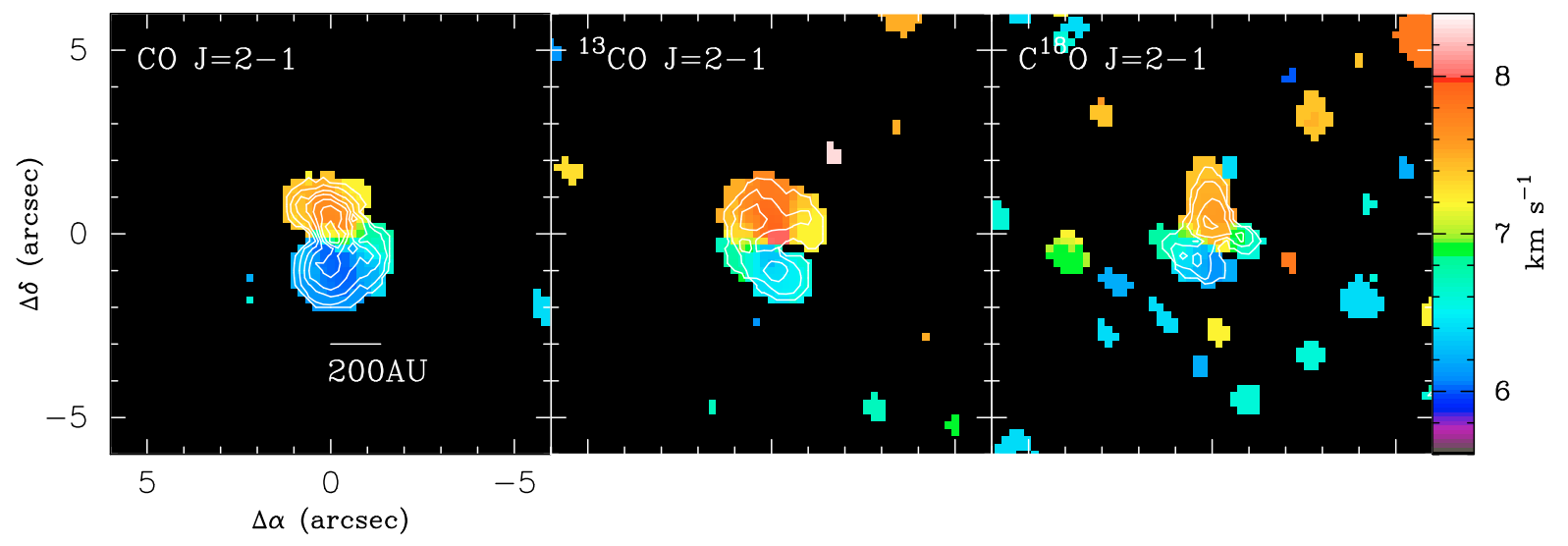

Fig. 1. Integrated intensity (contours) and first moment maps (colour scale) of ${ }^{12} \mathrm{CO} J=2-1$ (left panel, from Raman et al. 2006), ${ }^{13} \mathrm{CO} J=2-1$ (middle panel) and $\mathrm{C}^{18} \mathrm{O} J=2-1$ line (right panel). Contours are $1,2,3, \ldots \times 200 \mathrm{mJy} \mathrm{beam}^{-1} \mathrm{~km} \mathrm{~s}^{-1}$ for ${ }^{12} \mathrm{CO}$ and ${ }^{13} \mathrm{CO}$, and $1,2,3, \ldots \times$ $100 \mathrm{mJy} \mathrm{beam}^{-1} \mathrm{~km} \mathrm{~s}^{-1}$ for $\mathrm{C}^{18} \mathrm{O}$. The integrated intensity and first moment maps are obtained over a velocity range of $5.6-8.4 \mathrm{~km} \mathrm{~s}{ }^{-1}$. The data were clipped at $0.7,0.5$, and $0.35 \mathrm{Jy}_{\text {beam }}{ }^{-1}$ for ${ }^{12} \mathrm{CO},{ }^{13} \mathrm{CO}$, and $\mathrm{C}^{18} \mathrm{O}$, respectively.

Raman et al. (2006) spatially resolve the disc and find a fit to the CO $J=2-1$ line and $1.3 \mathrm{~mm}$ continuum observations by adopting a flared accretion disc model with a 235 AU radius and a $13^{\circ}$ inclination from face on. Observations at optical, IR, and (sub)millimetre wavelengths allowed modelling of the disc's spectral energy distribution (SED) (Malfait et al. 1998; Dominik et al. 2003; Dent et al. 2006). Malfait et al. (1998) fitted the nearinfrared and far-infrared excess of HD 169142 by two disc components: an inner disc extending from $0.5 \mathrm{AU}$ to $1 \mathrm{AU}$ with a density exponent of 2.0 and the outer disc from 28 AU with a flatter density distribution. Dominik et al. (2003) adopt a low inclination of $8^{\circ}$, outer radius of $100 \mathrm{AU}$, and surface density exponent $p=2$ to fit the SED, and therefore derive a disc mass of $0.1 M_{\odot}$. A more detailed SED modelling is done by Dent et al. (2006) where both the SED and resolved $7 \mathrm{~mm}$ continuum emission were fitted using an accretion disc model (D'Alessio et al. 2005) corresponding to a 10 Myr old A2 spectral type star. They adopt an inclination of $30^{\circ}$ and outer radius of $300 \mathrm{AU}$, and derive a disc mass of $4 \times 10^{-2} M_{\odot}$. Grady et al. (2007) fit the SED and NICMOS image at $1.1 \mu \mathrm{m}$ with a model consisting of two distinct disc components - the inner disc from 0.15 to $5 \mathrm{AU}$ radius and the outer disc extending from 44 to $230 \mathrm{AU}$. It is important to stress that all the above mass estimates of the disc around HD 169142 are based solely on the observed dust emission, and not gas.

This paper presents resolved interferometric observations of the ${ }^{13} \mathrm{CO}$ and $\mathrm{C}^{18} \mathrm{O} J=2-1$ lines from HD 169142. The observations and results are shown in Sects. 3 and 4. Section 4 introduces the disc model we adopt (D'Alessio et al. 2005; Dent et al. 2006; Raman et al. 2006) and our fit to the $1.3 \mathrm{~mm}$ data providing a measure of the minimum dust mass of the disc. We fit the ${ }^{13} \mathrm{CO}$ and $\mathrm{C}^{18} \mathrm{O}$ emission, thereby deriving the corresponding ${ }^{13} \mathrm{CO}$ and $\mathrm{C}^{18} \mathrm{O}$ mass in the disc. We place constraints on the total gas mass of the disc. We discuss the implications for the gas-to-dust ratio and micro-turbulence in the disc. Section 5 summarises our findings.

\section{Observations and results}

The observations of HD 169142 were carried out with the Submillimeter Array ${ }^{1}$ (SMA) on 2005 April 19, simultaneous

\footnotetext{
1 The Submillimeter Array is a joint project between the Smithsonian Astrophysical Observatory and the Academia Sinica Institute of
}

with the observations of ${ }^{12} \mathrm{CO} J=2-1$ line presented in Raman et al. (2006). A more detailed description of the observations and of the calibration procedure is given there. The correlator provided $2 \mathrm{GHz}$ of bandwidth in each sideband and was configured to include the ${ }^{13} \mathrm{CO} J=2-1$ line at $220.3986765 \mathrm{GHz}$ and the $\mathrm{C}^{18} \mathrm{O} J=2-1$ line at $219.5603568 \mathrm{GHz}$ in the lower sideband in a $104 \mathrm{MHz}$ wide spectral band with channel spacing of $0.2 \mathrm{MHz}\left(\sim 0.26 \mathrm{~km} \mathrm{~s}^{-1}\right)$.

The data reduction and image analysis were done with the Miriad data reduction tools (Sault et al. 1995). The $(u, v)$ data were Fourier transformed using natural weighting. The resulting synthesized beam size is $1^{\prime \prime} .4 \times 1^{\prime \prime} .0\left(\mathrm{PA}=26^{\circ}\right)$. The rms of the line images is $180 \mathrm{mJy}^{\text {beam }}{ }^{-1}$ per channel or $4.6 \mathrm{~K}(4.8 \mathrm{~K}$ for $\left.{ }^{12} \mathrm{CO}\right)$.

Emission of ${ }^{13} \mathrm{CO}$ and $\mathrm{C}^{18} \mathrm{O} J=2-1$ was detected from the HD 169142 circumstellar disc. Figure 1 shows the intensity weighted velocity maps with overlaid integrated intensity contours for both lines as well as the previously published ${ }^{12} \mathrm{CO} J=$ 2-1 line (Raman et al. 2006). The intensity integrated over the velocity range from 5.6 to $8.4 \mathrm{~km} \mathrm{~s}^{-1}$, in which the line emission is fully contained, and over the central $4^{\prime \prime} \times 4^{\prime \prime}$ region is 12.1 , 6.5 and $2.7 \mathrm{Jy} \mathrm{km} \mathrm{s}^{-1}$ for ${ }^{12} \mathrm{CO},{ }^{13} \mathrm{CO}$ and $\mathrm{C}^{18} \mathrm{O} J=2-1$ line, respectively. All three lines follow a similar velocity pattern, interpreted as a clear indication of Keplerian rotation around a $2 M_{\odot}$ star of a disc seen at $13^{\circ}$ inclination (Raman et al. 2006; this work). Figure 2 shows the ${ }^{12} \mathrm{CO},{ }^{13} \mathrm{CO}$ and $\mathrm{C}^{18} \mathrm{O} \mathrm{J}=2-1$ line spectra summed over a $4^{\prime \prime} \times 4^{\prime \prime}$ region centred on the HD 169142 position. The profiles are relatively symmetric, double-peaked, and centered on $7.1 \pm 0.2 \mathrm{~km} \mathrm{~s}^{-1}$ and reflecting the underlying rotation pattern. The ${ }^{12} \mathrm{CO}$ and $1.3 \mathrm{~mm}$ results were presented by Raman et al. (2006). They report a $1.3 \mathrm{~mm}$ line flux of $169 \pm$ $5 \mathrm{mJy}$. In Fig. 3 the emission stucture is shown over a range of velocities corresponding to the ${ }^{13} \mathrm{CO} J=2-1$ line (top panel). The emission extends to $2^{\prime \prime}$ ( $270 \mathrm{AU}$ ) from the star at a $2 \sigma$ level and shows a Keplerian velocity pattern. Figure 4 presents the channel maps of the $\mathrm{C}^{18} \mathrm{O} J=2-1$ line (top panel) with a kinematic structure similar to that of the ${ }^{13} \mathrm{CO} J=2-1$ line seen in Fig. 3. The $2 \sigma$ level emission reaches 1'.5 (220 AU) from the star. At $7.4 \mathrm{~km} \mathrm{~s}^{-1}$ a localised emission peak of $0.94 \pm 0.35 \mathrm{Jy}$ is seen 1 .'0 north from the star.

Astronomy and Astrophysics and is funded by the Smithsonian Institution and the Academia Sinica. 


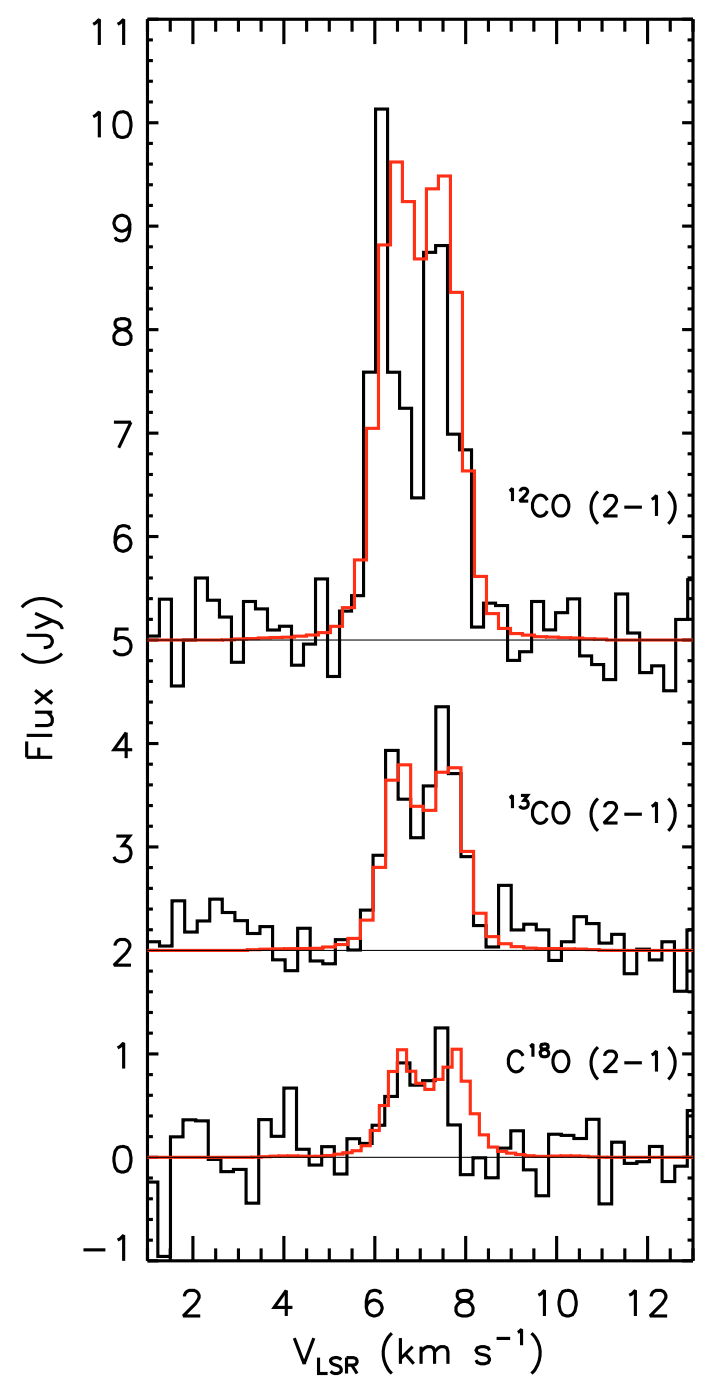

Fig. 2. Spectra of $\mathrm{C}^{18} \mathrm{O} J=2-1$ (bottom), ${ }^{13} \mathrm{CO} J=2-1$ (middle), and ${ }^{12} \mathrm{CO} J=2-1$ line (top, from Raman et al. 2006) summed over the central $4^{\prime \prime} \times 4$ " toward HD 169142. The ${ }^{13} \mathrm{CO}$ and ${ }^{12} \mathrm{CO}$ fluxes are shifted vertically by 2 and 5 Jy, respectively. Black lines show the observed spectra and the red lines show the model fit found in Sect. 4.3.1. For ${ }^{12} \mathrm{CO}$, the model from Raman et al. (2006) was used.

\section{Discussion}

Raman et al. (2006) show that the model that fits the SED of HD 169142 can also be used to fit the structure and intensity of the resolved ${ }^{12} \mathrm{CO}$ emission. They note that some weak residual emission is still present after subtracting the model from the observational data, that may correspond to real substructure within the disc. We analysed the ${ }^{13} \mathrm{CO}$ and $\mathrm{C}^{18} \mathrm{O}$ data, which are expected to probe different depths in the disc due to their lower opacities.

\subsection{Adopted disc model}

Dent et al. (2006) fit the spectral energy distribution (SED) of HD 169142 using an accretion disc model from the D'Alessio et al. (2005) database with an accretion rate of $10^{-8} M_{\odot} \mathrm{yr}^{-1}$, an outer radius of $300 \mathrm{AU}$, and a $30^{\circ}$ inclination. The age of the central star in this model is $10 \mathrm{Myr}$. They adopt an A2 spectral type for the central star because it provides a slightly better fit than the A6 spectral type model does to the SED in the range of 2-200 $\mu \mathrm{m}$ dominated by the warm dust emission. However, there is no significant difference between the SEDs corresponding to these two models. Beyond $44 \mathrm{AU}$ from the star, the model is qualitatively similar to the model of the outer disc of HD 169142 described in Grady et al. (2007). The detailed model structure, including plots of surface density distribution, temperature at different scale heights, and optical thickness of the disc, is provided in the online database of accretion disc models (D'Alessio et al. 2005) $)^{2}$.

In order to match their resolved submillimetre observations of CO, Raman et al. (2006) modify the model used in Dent et al. (2006) to have a disc radius of 235 AU. Assuming the mass of the central star to be $2 M_{\odot}$, they derive an inclination of $13 \pm$ $1^{\circ}$ from face-on and use it in the model. These modifications are not expected to affect the quality of the SED fit since the SED of HD 169142 alone does not constrain the outer radius and inclination of the disc well. They show that this SED model can be used to fit the structure and intensity of the resolved ${ }^{12} \mathrm{CO}$ emission. However, the SED does not provide reliable constraints of the disc dust mass, which in the model is set to $2 \times 10^{-4} M_{\odot}$. This parameter depends only on the optically thin thermal emission from the disc in the millimetre wavelength region.

The density in the model is a parameter that can be scaled by a factor of few, without significantly affecting the computed disc temperature structure. The resulting mass only affects the millimetre flux in the modelled SED, i.e., the $1.3 \mathrm{~mm}$ data point. In Sect. 4.2 we re-analyse the $1.3 \mathrm{~mm}$ data from Raman et al. (2006) using a radiative transfer code to calculate the emission from the model and better constrain the dust mass of the disc.

In the analysis of our ${ }^{13} \mathrm{CO}$ and $\mathrm{C}^{18} \mathrm{O}$ line emission (Sect. 4.3) we use this improved disc model, which already encompasses a range of observational constraints. We assume that the gas follows the dust distribution prescribed by the model and that their temperatures are the same. The micro turbulent FWHM of the lines is set to $0.16 \mathrm{~km} \mathrm{~s}^{-1}$ throughout the disc. One conclusion that we can already draw from the disc model is the fraction of disc mass where freeze-out of CO is likely to be efficient, which is believed to happen at temperatures below $20 \mathrm{~K}$. The mass weighted temperature in the model is $35 \mathrm{~K}$, which indicates that the bulk of the disc material is at temperatures above $20 \mathrm{~K}$. The temperature falls below $20 \mathrm{~K}$ only in the midplane region of the disc beyond $150 \mathrm{AU}$ from the star and there the abundance of the gas phase $\mathrm{CO}$ and its isotopologues is expected to be heavily decreased due to freeze-out of these molecules onto dust grains. This region contains only $8 \%$ of disc mass and therefore any mass estimate based on ${ }^{13} \mathrm{CO}$ and $\mathrm{C}^{18} \mathrm{O}$ emission may represent $92-100 \%$ of the true disc mass.

In all previously published work on HD 169142, the disc gas mass was inferred indirectly from the derived disc dust mass, adopting a standard gas-to-dust ratio. In our analysis of the ${ }^{13} \mathrm{CO}$ and $\mathrm{C}^{18} \mathrm{O}$ emission we argue that it is reasonable to assume a standard ${ }^{12} \mathrm{CO}$ abundance in the case of HD 169142, because freeze-out affects less than $8 \%$ of the mass in this disc. Therefore we investigate the constraints that can be obtained from our molecular line data on the disc gas mass.

\subsection{Dust continuum emission}

From the observed $1.3 \mathrm{~mm}$ continuum flux of $169 \pm 5 \mathrm{mJy}$, Raman et al. (2006) derive a dust mass of $2 \times 10^{-4} M_{\odot}$ assuming an emissivity $\kappa_{v}=2 \mathrm{~cm}^{2} \mathrm{~g}_{\text {dust }}^{-1}$ and a single temperature of $30 \mathrm{~K}$. We reanalyse the $1.3 \mathrm{~mm}$ emission using the disc

2 wWw.astrosmo.unam.mx/ dalessio/ 


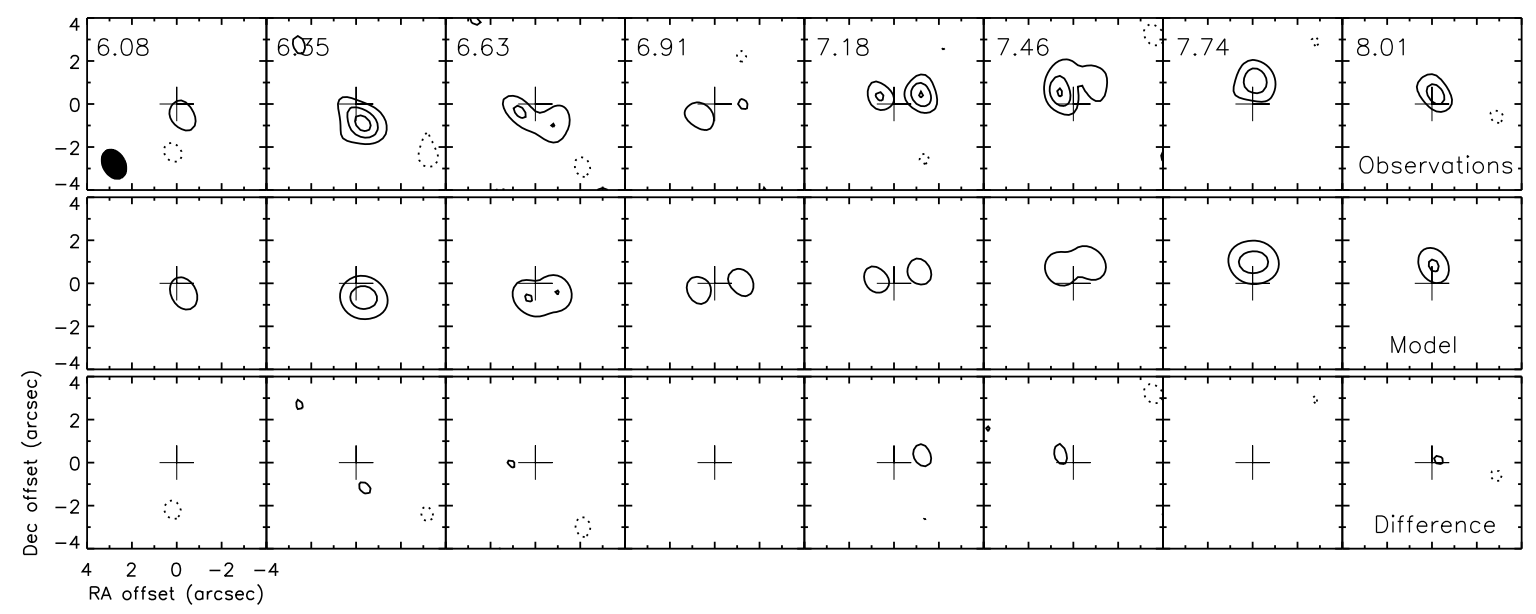

Fig. 3. Top panel: channel maps of the observed ${ }^{13} \mathrm{CO} J=2-1$ emission at a range of velocities over which the line is detected. The lower left corner of this panel shows the size and position angle of the synthesized beam. Middle panel: ${ }^{13} \mathrm{CO} J=2-1$ chanel maps from the best-fit disc model from Sect. 4.3.1. Lower panel: channel maps showing the residual emission after subtraction of the best-fit model from the data. The contour levels are $-1,1-4 \times 360 \mathrm{mJy}^{\text {beam }}{ }^{-1}(2$ sigma) in all panels.

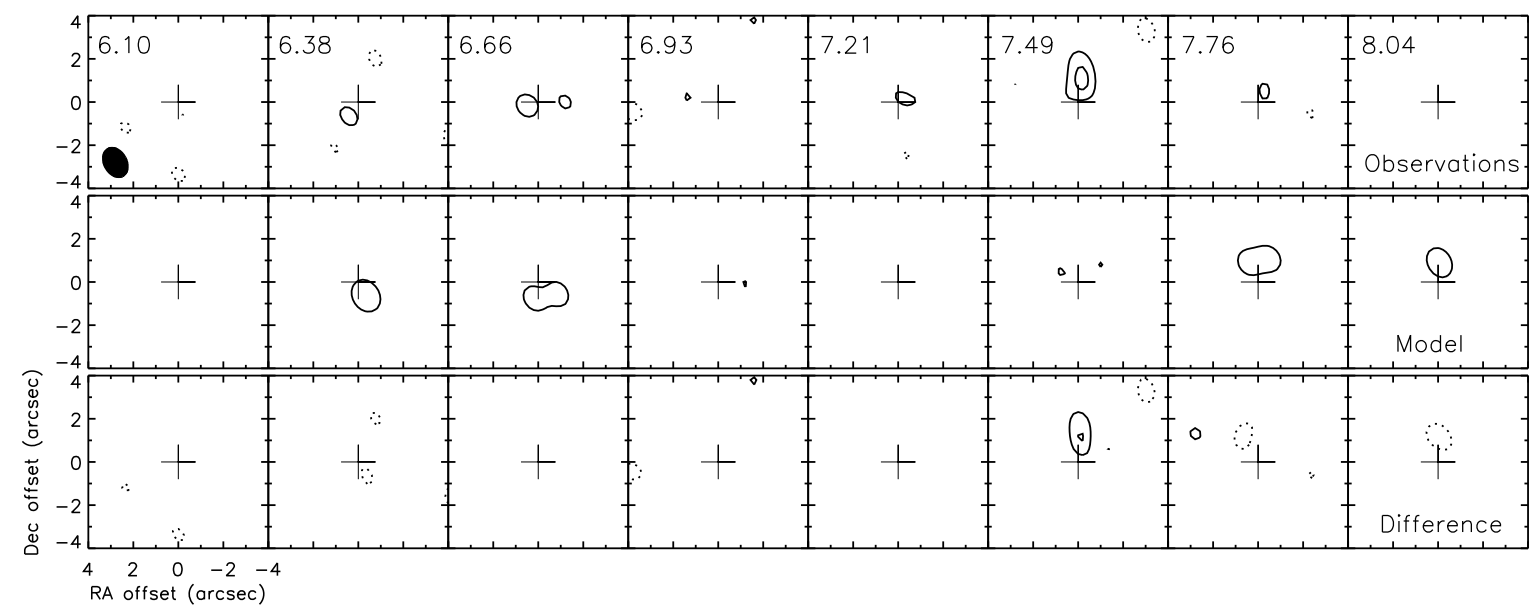

Fig. 4. Top panel: channel maps of the observed $\mathrm{C}^{18} \mathrm{O} J=2-1$ emission at a range of velocities over which the line is detected. The lower left corner of this panel shows the size and position angle of the synthesized beam. Middle panel: $\mathrm{C}^{18} \mathrm{O} J=2-1$ channel maps from the best-fit disc model from Sect. 4.3.1. Lower panel: channel maps showing the residual emission after subtraction of the best-fit model from the data. The contour levels are $-1,1-5 \times 360 \mathrm{mJy}^{\text {beam }}{ }^{-1}(2$ sigma $)$ in all panels.

temperature and density structure from the model described in Sect. 4.1, where we vary the dust density (i.e., the dust mass) to fit the observations. A large uncertainty on the mass determination from the $1.3 \mathrm{~mm}$ continuum flux is due to the dust opacity, which is not well-determined in circumstellar discs. Ossenkopf \& Henning (1994) study the effects of dust coagulation, and ice coverage of grains on their opacity in protostellar cores and suggest $1.3 \mathrm{~mm}$ opacity of $1 \mathrm{~cm}^{2} \mathrm{~g}_{\text {dust }}^{-1}$ for very dense $\left(n>10^{7} \mathrm{~cm}^{-3}\right.$ ) regions, and up to 5 times more if the grains are not covered in ice mantles. The latter is thus only valid for regions above water ice desorption temperature (80-100 K, Fraser et al. 2001). In their study of the dust opacity in circumstellar discs Draine (2006) find that astrosilicate and pirolysed cellulose at $600{ }^{\circ} \mathrm{C}$ are materials representative of the dust properties which may be expected in circumstellar discs. They explore a wide range of grain size distributions and find that these materials have mm-wavelength opacities which are close to the observational constraints on dust opacity from extinction studies of the diffuse interstellar medium presented in Weingartner \& Draine (2001). The resulting opacity could be anywhere between 0.1 and $2 \mathrm{~cm}^{2} \mathrm{~g}_{\text {dust }}^{-1}$ at the wavelength of $1.3 \mathrm{~mm}$, depending on the adopted grain size. We use this information to calculate the minimum amount of dust needed to produce the observed flux by adopting the opacity (and emissivity) of $2 \mathrm{~cm}^{2} \mathrm{~g}_{\text {dust }}^{-1}$. The SED modelling of the disc emission at shorter wavelengths is not affected by our assumptions of dust opacity at $1.3 \mathrm{~mm}$. The dust thermal emission is dominated by the cold disc midplane at large radii, likely to contain settled and/or grown dust particles whose optical properties may differ from those of the small dust at disc surface, responsible for the optically thick near-infrared and infrared emission of the disc.

We do the full modelling of the interferometric visibilities using the radiative transfer code of Hogerheijde \& van der Tak (2000, RATRAN). We perform a $\chi^{2}$ minimisation to fit the $1.3 \mathrm{~mm}$ visibilities by varying the disc dust mass. The best match is shown in Fig. 5, top-left panel, resulting in an estimate of the dust mass of the disc, given by

$M_{\text {dust }}=2.16 \times 10^{-2} \frac{2 \mathrm{~cm}^{2} \mathrm{~g}_{\text {dust }}^{-1}}{\kappa_{1.3 \mathrm{~mm}}} \times M_{\odot}$.

Assuming that the adopted dust opacity of $2 \mathrm{~cm}^{2} \mathrm{~g}_{\text {dust }}^{-1}$ is the maximum value, our estimate presents the lower limit on the 

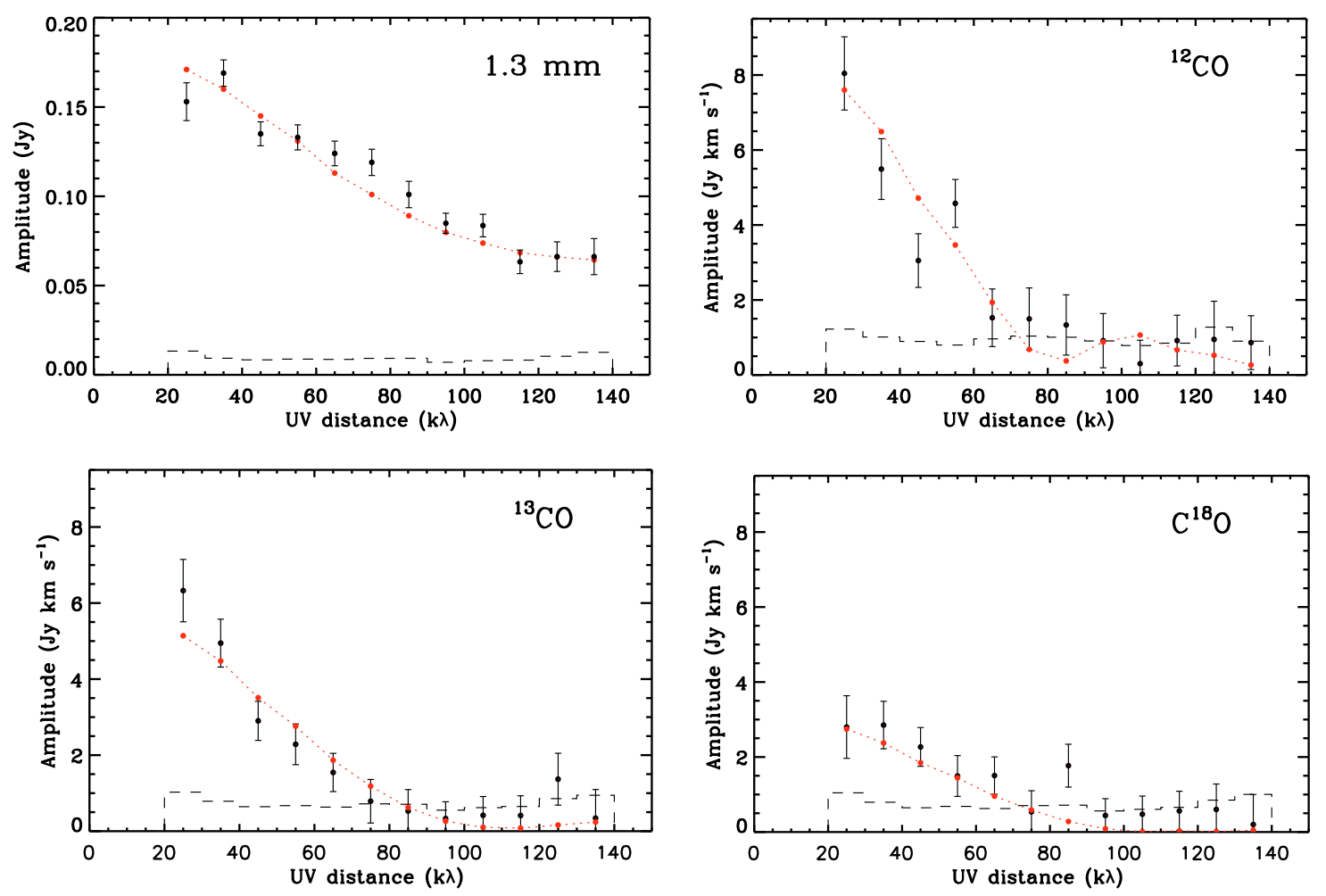

Fig. 5. Top-left: vector-averaged continuum flux as a function of projected baseline length (black dots). The error bars represent the variance within each annular average. The zero-signal expectation value is shown by the dashed histogram. The red dotted line shows the best-fit model for a dust emissivity of $2.0 \mathrm{~cm}^{2} \mathrm{~g}_{\text {dust }}^{-1}$ with the disc mass of $2.16 \times 10^{-4} M_{\odot}$. Top-right: same for the ${ }^{12} \mathrm{CO}$ emission from Raman et al. (2006), integrated over the width of $\approx 2.7 \mathrm{~km} \mathrm{~s}^{-1}$ (10 spectral channels) centred on each line. The red dotted line shows the model presented by Raman et al. (2006). Bottom-left and bottom-right: same for our ${ }^{13} \mathrm{CO}$ and $\mathrm{C}^{18} \mathrm{O} J=2-1$ data, respectively. The red dotted lines show the best-fit model from Sect. 4.3.1.

dust mass in HD 169142: $M_{\text {dust }} \geq 2.16 \times 10^{-4} M_{\odot}$. A slightly higher maximum dust opacity of $7 \mathrm{~cm}^{2} \mathrm{~g}_{\text {dust }}^{-1}$ is obtained for pyrolised cellulose at $800{ }^{\circ} \mathrm{C}$ but this value exceeds greatly the observational contraints from Weingartner \& Draine (2001) and although not impossible, is unlikely in discs (Draine 2006). However we cannot exclude the possibility that other dust properties like cristallinity, porosity and shape, which are not understood well, may enhance dust submillimetre opacity beyond $2 \mathrm{~cm}^{2} \mathrm{~g}_{\text {dust }}^{-1}$ by a factor of a few.

\subsection{Molecular line emission}

Due to their low abundance relative to ${ }^{12} \mathrm{CO}$, the ${ }^{13} \mathrm{CO}$ and $\mathrm{C}^{18} \mathrm{O}$ molecules emit lines that are comparatively less optically thick. As a result of this, they saturate at different heights in the disc allowing us to use them as a probe of the physical conditions in different disc layers and investigate the disc vertical structure (Dartois et al. 2003). Furthermore, the $\mathrm{C}^{18} \mathrm{O}$ line emission is often optically thin in the outer disc region, where the bulk of disc mass is located, which makes it a valuable probe of the disc gas mass.

The commonly employed route for the analysis of the $\mathrm{CO}$ isotopologue and dust continuum emission from circumstellar discs is to convert the continuum flux to disc mass by adopting a certain dust opacity, usually $0.02 \mathrm{~cm}^{2} \mathrm{~g}_{\text {gas }}^{-1}$ (Beckwith et al. 1990), under the assumption of the canonical gas-todust mass ratio of 100 . In this way, the molecular line emission is fit for abundance, where any discrepancy with respect to the canonical abundances and isotopic ratios of observed molecules is explained by a depletion factor, indicating the fraction of the $\mathrm{CO}$ gas affected by the freeze-out onto dust grains or selective photodissociation. This approach is used in Raman et al. (2006) where the analysis of the ${ }^{12} \mathrm{CO} J=2-1$ line emission and $1.3 \mathrm{~mm}$ continuum from HD 169142 is done for a disc mass of $2 \times 10^{-2} M_{\odot}$ and ${ }^{12} \mathrm{CO}$ was found to be depleted by a factor four, having an abundance of $2.5 \times 10^{-5}$. This corresponds to $6 \times 10^{-6} M_{\odot}$ of ${ }^{12} \mathrm{CO}$ molecules present in gas phase in the disc. In the present work, we argue that the ${ }^{12} \mathrm{CO}$ abundance is not significantly affected by freeze-out or selective photodissociation and we chose to follow a different route, in which we derive a rough gas mass estimate from the molecular line observations taking into account all related uncertainties.

To interpret our ${ }^{13} \mathrm{CO}$ and $\mathrm{C}^{18} \mathrm{O}$ observations, we adopt the disc model described in detail in Sect. 4.1. We set the dust mass in the model to $2.16 \times 10^{-4} M_{\odot}$; the value derived as the minimum dust mass for HD 169142 in Sect. 4.2. We assume that the molecular gas follows the dust temperature and density structure and we vary the ${ }^{13} \mathrm{CO}$ and $\mathrm{C}^{18} \mathrm{O}$ gas masses to fit the observations. We calculate the ${ }^{13} \mathrm{CO}$ and $\mathrm{C}^{18} \mathrm{O} J=2-1$ line emission from the model using the RATRAN molecular excitation and radiative transfer code. The model visibilities are generated with the Miriad task uvmodel, and the comparison to the observed visibilities is done directly. We use assumptions about the molecular abundances of the ${ }^{12} \mathrm{CO}$ and isotopic ratios to make a conversion to the total gas mass $\left(1.2 \times M_{\mathrm{H}_{2}}\right)$. We explore a range of values of the gas-to-dust mass ratio $f_{\mathrm{g} / \mathrm{d}}$.

\subsubsection{Disk structure probed by ${ }^{13} \mathrm{CO}$ and $\mathrm{C}^{18} \mathrm{O}$}

We find fits to the observed visibilities of ${ }^{13} \mathrm{CO}$ and $\mathrm{C}^{18} \mathrm{O}$ line for $M_{{ }^{13} \mathrm{CO}}=2.9 \times 10^{-7} M_{\odot}$ and $M_{\mathrm{C}^{18} \mathrm{O}}=4.6 \times 10^{-8} M_{\odot}$, shown in the bottom panels of Fig. 5. For comparison, the visibilities of 


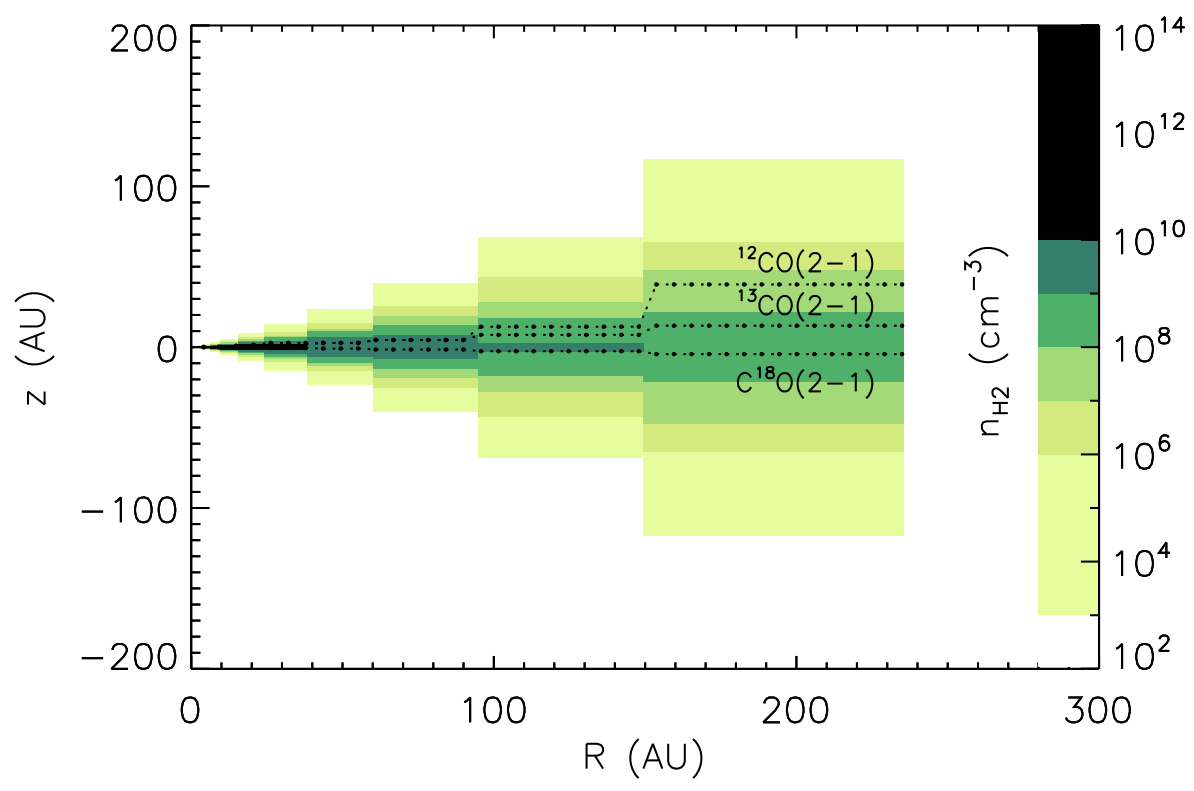

Fig. 6. Density structure of the best-fit disc model shown as colour scale. The disc is viewed by us from almost straight above. The three dotted lines show the heights above which $90 \%$ of the integrated line emission of ${ }^{12} \mathrm{CO}$, ${ }^{13} \mathrm{CO}$ and $\mathrm{C}^{18} \mathrm{O} J=2-1$ originates. For the $\mathrm{C}^{18} \mathrm{O}$ emission this line lies below the disc midplane. the ${ }^{12} \mathrm{CO} J=2-1$ line are also shown in Fig. 5, top-right panel, with the best fit obtained by Raman et al. (2006) for $M^{12} \mathrm{CO}=$ $6 \times 10^{-6} M_{\odot}$ contained in the gas phase. In order to test our fit further, we invert and deconvolve the visibilities using the miriad reduction package and compare the observed (top panels) and modelled (middle panels) velocity channel maps in Figs. 3 and 4. The structure and intensity of the emission is found to be well-matched. Figures 3 and 4 (lower panels) show the residual emission resulting from subtraction of the modelled from the observed data. For ${ }^{13} \mathrm{CO}$ the residuals appear randomly distributed and do not exceed the noise level. Significant residuals are seen in $\mathrm{C}^{18} \mathrm{O}$ at $7.4 \mathrm{~km} \mathrm{~s}^{-1}$ about 0.5 north from the star. The $\mathrm{C}^{18} \mathrm{O}$ data (Fig. 4) have a marginally significant localised excess 1 1.'0 north of the star seen at $3 \sigma$ level in the residual emission. This excess emission corresponds to the redshifted peak of the spectral line at $v_{\mathrm{LSR}}=7.4 \mathrm{~km} \mathrm{~s}^{-1}$. It is marginally resolved at $1^{\prime \prime} .5 \times 2$.' 5 and contains about $20 \%$ of $\mathrm{C}^{18} \mathrm{O}$ line flux. Similar, but not spatially coincident residual emission is also seen in ${ }^{13} \mathrm{CO}$ (Fig. 3, lower panel) near the noise levels, but not in ${ }^{12} \mathrm{CO}$. A cause of this feature may lie in disc midplane asymmetry. Any connection with the reported companion at 9.'3 is unlikely, but a hypothesized body within the inner disc is an interesting prospect (Grady et al. 2007). Nevertheless, an instrumental or calibration artefact may have affected the $7.4 \mathrm{~km} \mathrm{~s}^{-1}$ channel resulting in a slight increase of flux in that channel in the lower sideband, only noticeable in the weak $\mathrm{C}^{18} \mathrm{O}$ line.

In this section we will use our ${ }^{13} \mathrm{CO}$ and $\mathrm{C}^{18} \mathrm{O}$ results to make an estimate of the total gas mass $M_{\text {gas }}$ in the disc, related to the ${ }^{12} \mathrm{CO}$ gas mass $M^{12} \mathrm{CO}$ through

$M_{\mathrm{gas}}=1.2 \times \frac{m_{\mathrm{H}_{2}}}{m_{{ }^{12} \mathrm{CO}}} \times \frac{1}{\left[{ }^{12} \mathrm{CO}\right]} \times M_{{ }^{12} \mathrm{CO}}$

where $m_{\mathrm{H}_{2}}$ and $m_{12} \mathrm{CO}$ are the masses of the $\mathrm{H}_{2}$ and ${ }^{12} \mathrm{CO}$ molecule, and $\left[{ }^{12} \mathrm{CO}\right]$ is the abundance of ${ }^{12} \mathrm{CO}$ with respect to $\mathrm{H}_{2}$. The factor of 1.2 accounts for one fifth of the gas contained in helium. We can use the derived masses of the ${ }^{13} \mathrm{CO}$ and $\mathrm{C}^{18} \mathrm{O}$ gas to calculate the mass of the ${ }^{12} \mathrm{CO}$ gas. We adopt the isotopic ratios of ${ }^{12} \mathrm{C} /{ }^{13} \mathrm{C}=77 \pm 7$ and ${ }^{16} \mathrm{O} /{ }^{18} \mathrm{O}=560 \pm 25$ from Wilson \& Rood (1994).

$M^{12} \mathrm{CO}=\frac{m_{12} \mathrm{CO}}{m_{\mathrm{C}^{18} \mathrm{O}}} \times \frac{\left[{ }^{16} \mathrm{O}\right]}{\left[{ }^{18} \mathrm{O}\right]} \times M_{\mathrm{C}^{18} \mathrm{O}}=(2.4 \pm 1.0) \times 10^{-5} M_{\odot}$
$M_{{ }^{12} \mathrm{CO}}=\frac{m_{12} \mathrm{CO}}{m_{13} \mathrm{CO}} \times \frac{\left[{ }^{12} \mathrm{C}\right]}{\left[{ }^{13} \mathrm{C}\right]} \times M^{13} \mathrm{CO}=(2.2 \pm 0.6) \times 10^{-5} M_{\odot}$.

The values obtained here are given with errors which are dominated by the rms of our data and also include the errors on isotopic ratios. If we take into account that $8 \%$ of the ${ }^{12} \mathrm{CO}$ and its isotopologues in HD 169142 may be depleted onto dust grains and therefore not contributing to the emission observed, then the full ${ }^{12} \mathrm{CO}$ mass range is $(1.4-3.7) \times 10^{-5} M_{\odot}$. The fact that both datasets roughly agree on the ${ }^{12} \mathrm{CO}$ mass provides an additional argument for the validity of the model. However, the fit to the ${ }^{12} \mathrm{CO} J=2-1$ data done in Raman et al. (2006) corresponds to a mass of gas phase ${ }^{12} \mathrm{CO}$ of $6 \times 10^{-6} M_{\odot}$ (mentioned in Sect. 4.3), two to six times lower than the range derived here. The ${ }^{12} \mathrm{CO}$ emission is optically thick and therefore it does not trace the entire disc but originates from a layer much higher in the disc than either the ${ }^{13} \mathrm{CO}$ or $\mathrm{C}^{18} \mathrm{O}$ lines (Dartois et al. 2003). The discrepancy between the ${ }^{12} \mathrm{CO}$ result and that of the isotopologues indicates that the adopted disc model may not provide the best description in these high layers. We will discuss this in more detail in Sect. 4.5 where the effects of temperature and turbulence on the line profile are discussed.

Our model calculations allow us to identify which regions of the disc contribute the most to the observed lines. We followed the line-of-sight integration step by step from the observer into the disc and find the location where $90 \%$ of the integrated intensity is reached for a face-on orientation of the disc. We show these locations in Fig. 6 in a cross-section of the disc showing the underlying density structure. We chose to use the intensity integrated over a range of wavelengths in which the line is emitted, in order to better represent the region corresponding to the physical quantity that is observed, i.e., the line flux. The use of surfaces with equal opacity (often used $\tau=3$ ) may be misleading, because the opacity at the line centre is not necessarily consistent with the opacity in the line wings, and the amount of flux still produced in the line wings beyond the $\tau=3$ surface may contribute significantly to the line flux in some species more than in the others. The calculation was done for the ${ }^{12} \mathrm{CO},{ }^{13} \mathrm{CO}$, and $\mathrm{C}^{18} \mathrm{O} J=2-1$ lines. The discontinuous shape of the surface above which $90 \%$ of the integrated intensity in these lines is emitted stems from the discrete cells in the grid of our model that were used to sample the density and temperature structure. As 
Table 1. Column of ${ }^{12} \mathrm{CO}$ traced by $90 \%$ of the emission of each line, and the corresponding average temperature, at two radial intervals beyond $100 \mathrm{AU}$.

\begin{tabular}{c|cc|cccc|cc}
\hline \hline $\begin{array}{c}\text { Molecular } \\
\text { Line }\end{array}$ & $\begin{array}{c}z(\mathrm{AU}) \\
100-149 \mathrm{AU}\end{array}$ & $\begin{array}{c}z(\mathrm{AU}) \\
149-235 \mathrm{AU}\end{array}$ & $\begin{array}{c}N_{\mathrm{CO}}\left(\mathrm{cm}^{-2}\right) \\
100-149 \mathrm{AU}\end{array}$ & $\begin{array}{c}\text { Fraction Of } \\
\text { Total } N_{\mathrm{CO}}\end{array}$ & $\begin{array}{c}N_{\mathrm{CO}}\left(\mathrm{cm}^{-2}\right) \\
149-235 \mathrm{AU}\end{array}$ & $\begin{array}{c}\text { Fraction Of } \\
\text { Total } N_{\mathrm{CO}}\end{array}$ & $\begin{array}{c}T_{\text {ave }}(\mathrm{K}) \\
100-149 \mathrm{AU}\end{array}$ & $\begin{array}{c}T_{\text {ave }}(\mathrm{K}) \\
149-235 \mathrm{AU}\end{array}$ \\
\hline${ }^{12} \mathrm{CO} J=2-1$ & 12.7 & 39.0 & $6.5 \times 10^{17}$ & $7 \%$ & $6.6 \times 10^{16}$ & $1 \%$ & 33.3 & 38.6 \\
${ }^{13} \mathrm{CO} J=2-1$ & 7.6 & 13.0 & $4.2 \times 10^{18}$ & $17 \%$ & $2.6 \times 10^{18}$ & $18 \%$ & 29.1 & 25.6 \\
$\mathrm{C}^{18} \mathrm{O} J=2-1$ & -2.5 & -4.3 & $1.5 \times 10^{19}$ & $62 \%$ & $8.9 \times 10^{18}$ & $62 \%$ & 25.7 & 21.8 \\
\hline
\end{tabular}

expected, the less optically thick species trace regions deeper in the disc with $\mathrm{C}^{18} \mathrm{O}$ tracing the disc midplane, while ${ }^{12} \mathrm{CO}$ traces the disc surface layer. We analysed the physical properties of the emitting regions in the outer disc, beyond $100 \mathrm{AU}$ from the star (scales resolved by our SMA observations). For the two outermost radial positions in our model grid, we calculated the column of ${ }^{12} \mathrm{CO}$ gas contained in the region where $90 \%$ of the emission is coming from, which is located above the vertical position shown in Fig. 6, for each molecular line. These values are shown in Table $1^{3}$. Also, the average temperature of these gas columns was calculated, taking the temperature weighted by the column density (i.e., mass) into account for each cell located above the vertical positions from Fig. 6 . The $\mathrm{C}^{18} \mathrm{O} J=2-1$ line generally traces three times as much $\mathrm{CO}$ gas as traced by the ${ }^{13} \mathrm{CO} \mathrm{J}=$ $2-1$ line in the outer disc, while the ${ }^{12} \mathrm{CO} J=2-1$ line traces only a small fraction of the gas, a few percent of the column traced by $\mathrm{C}^{18} \mathrm{O}$. It is clearly seen that the $\mathrm{C}^{18} \mathrm{O}$ traces deep into the disc, to the height of several $\mathrm{AU}$ at the far side of the midplane, showing that it is very sensitive to the total gas mass. The $90 \%$ of the $\mathrm{C}^{18} \mathrm{O} J=2-1$ line emission traces around $60 \%$ of the total gas column.

In Sect. 4.1 we already concluded from the disc model that the $\mathrm{CO}$ freeze-out could only be efficient in the cold outer disc midplane region that contains $8 \%$ of the total disc mass. This very small fraction is due to the luminosity of the Herbig Ae star HD 169142, which is higher than the typical luminosity of $\mathrm{T}$ Tauri stars. In the latter, the circumstellar discs are colder and the $\mathrm{CO}$ freeze-out presents a major obstacle to determining gas mass based on observations of $\mathrm{CO}$ or its isotopologues. As a caveat, we note that Piétu et al. (2006) find somewhat lower dust temperature in the disc around the Herbig Ae star MWC 480 (10-25 K at $100 \mathrm{AU})$. Since we have no indications to the contrary, we adopt the dust temperature that SED disc modelling suggests. If observed with a sufficiently high resolution and sensitivity, discs where $\mathrm{CO}$ freeze-out is efficient are expected to have smaller radii derived from $\mathrm{CO}$ isotopologues compared to the outer radius derived from the ${ }^{12} \mathrm{CO}$ and from the dust emission. Although our observations are limited by the beam size $\left(1{ }^{\prime \prime} 4 \approx 200 \mathrm{AU}\right)$ we have tested a range of different outer radii and found that, based on our molecular line data, the outer radius should not be very different from 200 AU for either of the observed lines. We also do not find any significant discrepancy between the molecular line data and dust continuum that could indicate different outer radii for gas and dust.

Another factor that can influence the ${ }^{12} \mathrm{CO}$ abundance is photodissociation. The effect of photodissociation via the stellar UV radiation on $\mathrm{CO}$ abundance in circumstellar discs is studied in van Zadelhoff (2002) and van Zadelhoff et al. (2003). They find that the $\mathrm{CO}$ abundance decrease due to photodissociation is significant only in the upper disc layers and that the $\mathrm{CO}$ column density is not much affected by it. Jonkheid et al. (2004)

$\overline{3}$ The calculations for ${ }^{12} \mathrm{CO} J=2-1$ are done for the model presented in Raman et al. (2006). show that the stellar UV field is only able to heat $10 \%$ of the gas mass in the disc to considerably high temperatures, in the surface disc layer. The effect of photodissociation on the $\mathrm{CO}$ content of HD 169142 is expected to be small, due to the significant dust mass of the disc. Since we adopt a maximum emissivity and therefore minimal dust mass, the conclusion that photoionization can be neglected is robust. In addition, the $\mathrm{C}^{18} \mathrm{O}$ emission that we use to derive the gas mass, arises predominantly from the outer disc midplane region that contains the bulk of the disc mass and does not receive any direct UV illumination from the central star. This further minimises the effects of photodissociation on our results. Therefore, we find it appropriate to use the ${ }^{12} \mathrm{CO}$ mass derived from our best fit to the $\mathrm{C}^{18} \mathrm{O}$ and ${ }^{13} \mathrm{CO}$ data to make an estimate of the total gas mass.

The abundance of ${ }^{12} \mathrm{CO}$ is a key factor in this conversion and the values found in the literature for the interstellar medium range from $9.5 \times 10^{-4}$ (Frerking et al. 1982) to $2.0 \times 10^{-4}$ (Lacy et al. 1994). If we adopt the ${ }^{12} \mathrm{CO}$ abundance of $10^{-4}$ with respect to $\mathrm{H}_{2}$, we obtain $M_{\text {gas }}=1.2 \times M_{\mathrm{H}_{2}}=\left(2.0_{-0.8}^{+1.0}\right) \times 10^{-2} M_{\odot}$. The error estimate includes the error due to the rms of our $\mathrm{C}^{18} \mathrm{O}$ data $(41 \%)$ and to the isotopic ratio of $\left[{ }^{16} \mathrm{O}\right] /\left[{ }^{18} \mathrm{O}\right](4 \%)$. The upper margin is extended to include the $8 \%$ uncertainty in the gas mass estimate due to freeze-out. The factor 1.2 takes the gas mass contained in helium into account. Adopting a ${ }^{12} \mathrm{CO}$ abundance of $2.0 \times 10^{-4}$ with respect to $\mathrm{H}_{2}$ results in $M_{\text {gas }}$ as low as $6 \times 10^{-3} M_{\odot}$. Therefore, due to the uncertainty largely dominated by the ${ }^{12} \mathrm{CO}$ abundance, we can only conclude that the gas mass of the disc around HD 169142 falls in the range of $(0.6-3.0) \times 10^{-2} M_{\odot}$.

\subsection{Gas-to-dust ratio}

To investigate the gas-to-dust ratio, we ran several models in which we assume $M_{\text {dust }}=2.16 \times 10^{-4} M_{\odot},[\mathrm{CO}]=10^{-4}$, ${ }^{12} \mathrm{C} /{ }^{13} \mathrm{C}=77,{ }^{16} \mathrm{O} /{ }^{18} \mathrm{O}=560$, and gas-to-dust mass ratios of 100 , 50,33 , and 25 . From the comparison of the synthetic visibilities of these models with the observations (Fig. 7, middle and lower panel) we find that a good fit for ${ }^{13} \mathrm{CO}$ and $\mathrm{C}^{18} \mathrm{O}$ data is obtained for $f_{\mathrm{g} / \mathrm{d}}=100$. Under the assumptions used above, lower values of $f_{\mathrm{g} / \mathrm{d}}$ fail to provide a fit to the data. The ${ }^{12} \mathrm{CO}$ data (Fig. 7, top panel) shows a discrepancy, already discussed in Sect. 4.3.1, with respect to the ${ }^{13} \mathrm{CO}$ and $\mathrm{C}^{18} \mathrm{O}$ data in the sense that the model we use overestimates the ${ }^{12} \mathrm{CO}$ emission. This suggests that the model assumptions like the temperature, micro turbulence, and ${ }^{12} \mathrm{CO}$ abundance used for the upper disc layers where the ${ }^{12} \mathrm{CO}$ emission arises from should be reconsidered. From the gas and dust masses derived in Sects. 4.2 and 4.3, we see that a gas-to-dust mass ratio of 100:1 only holds for special circumstances considered here: a maximum dust emissivity of $2 \mathrm{~cm}^{2} \mathrm{~g}_{\text {dust }}^{-1}$ at $1.3 \mathrm{~mm}$ and a ${ }^{12} \mathrm{CO}$ abundance of $10^{-4}$ with respect to $\mathrm{H}_{2}$, or lower. For equally likely values of $1 \mathrm{~cm}^{2} \mathrm{~g}_{\text {dust }}^{-1}$ for the dust emissivity and $2 \times 10^{-4}$ for the ${ }^{12} \mathrm{CO}$ abundance, a gas-to-dust mass ratio of 25 would be inferred, suggesting that 


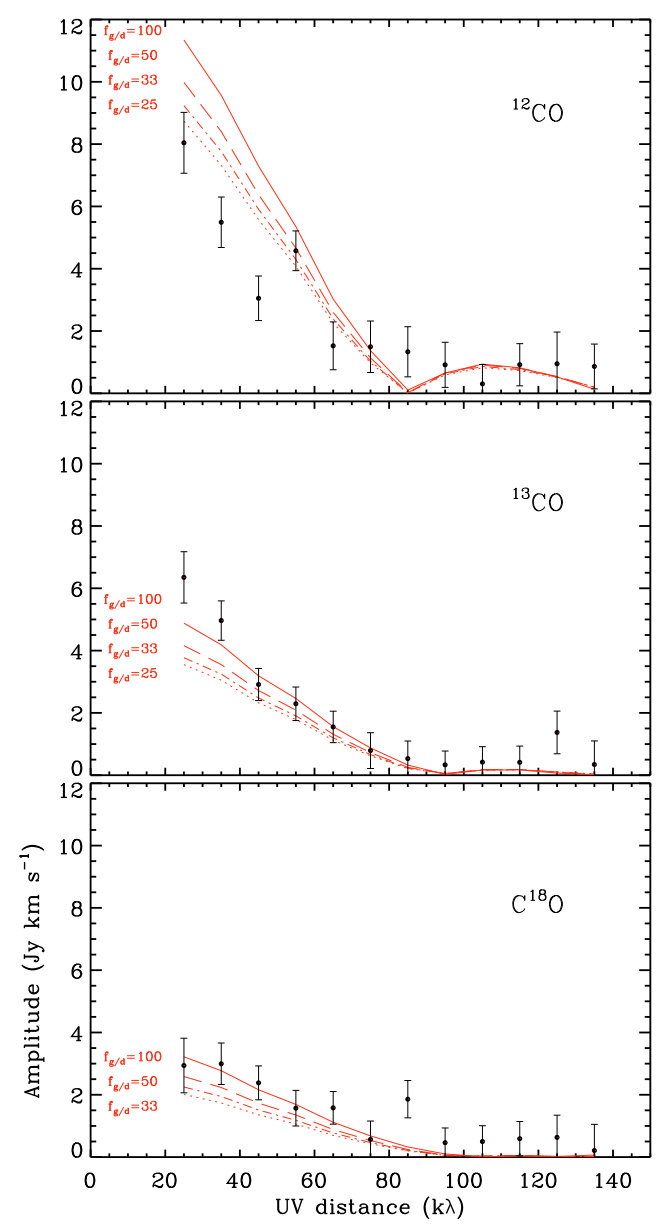

Fig. 7. Comparison of the observed vector-averaged ${ }^{12} \mathrm{CO}$ (top panel), ${ }^{13} \mathrm{CO}$ (middle panel) and $\mathrm{C}^{18} \mathrm{O}$ (lower panel) line fluxes (black) to four models with different gas-to-dust ratios (red lines). The error bars represent the variance within each annular average. The models assume the dust mass of $2.16 \times 10^{-4} M_{\odot}$ and gas-to-dust mass ratio $f_{\mathrm{g} / \mathrm{d}}=100,50$, 33,25 marked respectively by the full, dashed, dashed-dotted and dotted red lines in each panel. The line fluxes are integrated over the width of $\approx 2.7 \mathrm{~km} \mathrm{~s}^{-1}$ (10 spectral channels) centred on each line.

the disc has lost significant amounts of gas with respect to dust. In fact, gas loss is expected in circumstellar discs due to photoevaporation of gas by the stellar radiation. The typical timescale for this process of a few Myr (Hollenbach et al. 1994; Font et al. 2004) is similar to the estimated age of HD 169142 of $6_{-3}^{+6} \mathrm{Myr}$ (Grady et al. 2007).

\subsection{Micro turbulence}

As mentioned in the previous section, the overestimate of the ${ }^{12} \mathrm{CO}$ emission by the model that fits the ${ }^{13} \mathrm{CO}$ and $\mathrm{C}^{18} \mathrm{O} \mathrm{J}=$ 2-1 line emission well may be due to model assumptions used for the upper disc layers, e.g., temperature, ${ }^{12} \mathrm{CO}$ relative abundance, turbulence.

In Sect. 4.1 we mention that a disc model with a less luminous A6 spectral type star also reproduces the SED of HD 169142 well (Dent et al. 2006). However, the temperature in that model is less than $10 \%$ lower than the temperature used in our model. This is too low to provide a significant decrease in the ${ }^{12} \mathrm{CO}$ line flux.

A fit to all observed lines simultaneously by varying the isotopic ratios yields an unusually low value of both the ${ }^{12} \mathrm{C} /{ }^{13} \mathrm{C}$ and the ${ }^{16} \mathrm{O} /{ }^{18} \mathrm{O}$ isotopic ratios: 21 and 171 , respectively. These values are far from the observationally derived ratios in the interstellar medium and this scenario would be highly unlikely. An overabundance of ${ }^{13} \mathrm{CO}$ was measured at large radial distances from the star (several hundreds of AU) in the discs DM Tau, MWC 480, and LkCa 15 (Piétu et al. 2007). In general, discs are often found to have ${ }^{13} \mathrm{CO}$ and $\mathrm{C}^{18} \mathrm{O}$ abundances depleted by large factors, mainly in discs around T Tauri stars (Dutrey et al. 1994, 1996).

In the above analysis, the turbulent line $F W H M$ was set to $0.16 \mathrm{~km} \mathrm{~s}^{-1}$ throughout the disc. In a more realistic scenario, the turbulence in the cold and dense midplane differs from the turbulence in the warmer and less dense surface layers. We investigate the effect of micro turbulence on the modelled line emission and find that a simultaneous match is possible in our model, as shown in Fig. 8. This fit is obtained for a gas-to-dust ratio of 33, i.e., a disc gas mass of $7 \times 10^{-3} M_{\odot}$, if a higher value of turbulence is assumed for the disc midplane (turbulent FWHM of $0.33 \mathrm{~km} \mathrm{~s}^{-1}$ ) and no turbulence for the upper disc layers traced by the ${ }^{12} \mathrm{CO}$ emission $\left(0.00 \mathrm{~km} \mathrm{~s}^{-1}\right.$, only thermal broadening). For the ${ }^{12} \mathrm{CO} J=2-1$ line, that is fully optically thick at all radii in our model, the turbulent line broadening is degenerate with temperature and density in its effect on the line flux and profile. This is because the turbulence additionally broadens the thermally broadened line profile and in this way the optical thickness decreases allowing for more gas column to be traced. On the other hand, by decreasing the turbulence, at a given temperature, the same ${ }^{12} \mathrm{CO}$ data can be fit with comparably more $M^{{ }^{12} \mathrm{CO}}$. The effect of the increase in turbulence on the ${ }^{13} \mathrm{CO}$ and $\mathrm{C}^{18} \mathrm{O}$ lines is present but less pronounced, due to their lower optical thickness. In particular, the $\mathrm{C}^{18} \mathrm{O} J=2-1$ line emission is only marginally optically thick. The effect of turbulence on the modelled $\mathrm{C}^{18} \mathrm{O} J=2-1$ line emission is below the rms of our observations. It is not possible to obtain simultaneous fits to all data if the gas-to-dust ratio is higher than 33 (disc mass higher than $7 \times 10^{-3} M_{\odot}$ ). A lower gas-to-dust ratio (disc mass lower than $7 \times 10^{-3} M_{\odot}$ ) would require the turbulent $F W H M$ of the lines to be more than $0.33 \mathrm{~km} \mathrm{~s}^{-1}$ in the disc midplane.

\section{Summary}

The circumstellar disc around the Herbig Ae star HD 169142 was imaged in ${ }^{13} \mathrm{CO}$ and $\mathrm{C}^{18} \mathrm{O} J=2-1$ line emission at 1 .' 4 resolution. The disc is resolved and the emission extends to 200-300 AU from the star at 2 $\sigma$ levels. We adopted an accretion disc model that matches the SED of HD 169142 and we used a molecular excitation and radiative transfer code to analyse our data. We revisited existing $1.3 \mathrm{~mm}$ and ${ }^{12} \mathrm{CO}$ data and interpret it in view of our new results. The observed kinematic pattern and line profiles are consistent with a disc seen at $13^{\circ}$ inclination from face on, in Keplerian rotation around a $2 M_{\odot}$ star. The structure of the emission suggests a disc radius of roughly $200 \mathrm{AU}$ for both gas and dust.

- We calculated the minimum disc dust mass of $M_{\text {dust }}=2.16 \times$ $10^{-4} M_{\odot}$ through the fit to the $1.3 \mathrm{~mm}$ data using the maximum dust opacity theoretically predicted in circumstellar discs.

- The adopted disc model reproduces our molecular line observations with $M_{\mathrm{C}^{18} \mathrm{O}}=4.6 \times 10^{-8} M_{\odot}$ and $M_{13} \mathrm{CO}=2.9 \times$ $10^{-7} M_{\odot}$. The derived amount of $\mathrm{C}^{18} \mathrm{O}$ implies a $M_{\mathrm{CO}}=$ $(2.4 \pm 1.0) \times 10^{-5} M_{\odot}$. The ${ }^{13} \mathrm{CO}$ amount is also in agreement with this value. 


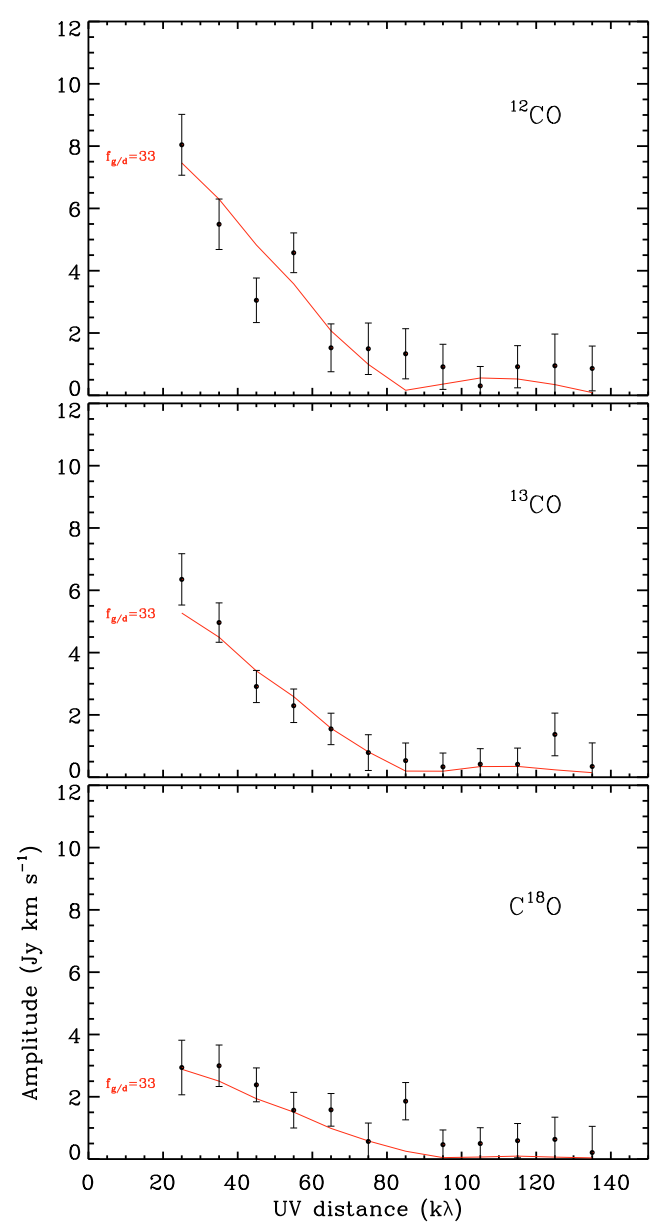

Fig. 8. Comparison of the observed vector-averaged ${ }^{12} \mathrm{CO}$ (top panel), ${ }^{13} \mathrm{CO}$ (middle panel) and $\mathrm{C}^{18} \mathrm{O}$ (lower panel) line fluxes (black) to a model which assumes the total disc mass of $7 \times 10^{-3} M_{\odot}$, gas-to-dust mass ratio $f_{\mathrm{g} / \mathrm{d}}=33$, no microturbulent line broadening in the upper disc layers from which the ${ }^{12} \mathrm{CO}$ emission originates (only thermal broadening), and a microturbulent broadening of $0.33 \mathrm{~km} \mathrm{~s}^{-1} F W H M$ in the layers from which the ${ }^{13} \mathrm{CO}$ and $\mathrm{C}^{18} \mathrm{O}$ emission arises.

- We identify the regions of the disc that contribute most to the emission of ${ }^{12} \mathrm{CO},{ }^{13} \mathrm{CO}$, and $\mathrm{C}^{18} \mathrm{O} J=2-1$ line and find, as expected due to different optical depth of these lines, that they are physically distinct. In particular, the $\mathrm{C}^{18} \mathrm{O} J=$ $2-1$ line is marginally optically thin throughout the disc and traces the bulk of the mass contained in the disc's midplane.

- We find that the effect of freeze-out in the model is relatively small, allowing us to make an estimate of the gas mass based on our observations. For plausible $\mathrm{CO}$ abundances of $(1-2) \times 10^{-4}$, we can only derive a range of $M_{\text {gas }}=$ $(0.6-3.0) \times 10^{-2} M_{\odot}$.

- The comparison of the derived gas and dust mass shows that the gas-to-dust mass ratio of 100 is only possible under the assumption of a high dust opacity of $2 \mathrm{~cm}^{2} \mathrm{~g}_{\text {dust }}^{-1}$ and low ${ }^{12} \mathrm{CO}$ abundance of $10^{-4}$ with respect to $\mathrm{H}_{2}$. Otherwise, this ratio may be as low as 25 .

- Existing observations of the optically thick ${ }^{12} \mathrm{CO}$ line can be fit simultaneously to our ${ }^{13} \mathrm{CO}$ and $\mathrm{C}^{18} \mathrm{O}$ data by the model adopted here, only if the micro turbulence in the disc midplane is higher than the micro turbulence in the upper disc layers that dominate the ${ }^{12} \mathrm{CO}$ emission.

The discrepancy between the $\mathrm{CO}$ gas mass derived here and the one derived based on the ${ }^{12} \mathrm{CO}$ line emission likely implies that the micro turbulence is much lower than $0.1 \mathrm{~km} \mathrm{~s}^{-1}$ in the upper disc layers where the ${ }^{12} \mathrm{CO}$ emission originates. Better constraints of the ${ }^{12} \mathrm{CO}$ abundance in discs are needed to enable us to measure gas mass more precisely in discs. Better knowledge of dust properties such as dust size distribution and composition is essential for a better estimate of dust opacity in discs. However, these properties vary from source to source and are not necessarily linked with other parameters, age or mass for example, and the studies need to focus on individual sources. Since the discs around Herbig Ae stars have the advantage that the gas mass estimate from $\mathrm{C}^{18} \mathrm{O}$ line emission is more reliable, it would be useful to investigate dust properties, i.e., emissivity in these sources in more detail, to be able to have a reliable estimate of dust mass as well. This would, for the first time, open a real opportunity to study the gas-to-dust mass ratio, the unknown property on which the theories of disc evolution and planet formation depend strongly.

Acknowledgements. The research of O.P. is supported through Marie Curie FP6 programme of the European Union. The research of O.P. and M.R.H. is supported through a VIDI grant from the Netherlands Organisation for Scientific Research. We would like to thank E. F. van Dishoeck for useful discussions.

\section{References}

Beckwith, S. V. W., Sargent, A. I., Chini, R. S., \& Guesten, R. 1990, AJ, 99, 924 D’Alessio, P., Merín, B., Calvet, N., Hartmann, L., \& Montesinos, B. 2005, Rev. Mex. Astron. Astrofis., 41, 61

Dartois, E., Dutrey, A., \& Guilloteau, S. 2003, A\&A, 399, 773

Dent, W. R. F., Greaves, J. S., \& Coulson, I. M. 2005, MNRAS, 359, 663

Dent, W. R. F., Torrelles, J. M., Osorio, M., Calvet, N., \& Anglada, G. 2006, MNRAS, 365, 1283

Dominik, C., Dullemond, C. P., Waters, L. B. F. M., \& Walch, S. 2003, A\&A, 398,607

Draine, B. T. 2006, ApJ, 636, 1114

Dutrey, A., Guilloteau, S., \& Simon, M. 1994, A\&A, 286, 149

Dutrey, A., Guilloteau, S., Duvert, G., et al. 1996, A\&A, 309, 493

Font, A. S., McCarthy, I. G., Johnstone, D., \& Ballantyne, D. R. 2004, ApJ, 607, 890

Fraser, H. J., Collings, M. P., McCoustra, M. R. S., \& Williams, D. A. 2001, MNRAS, 327, 1165

Frerking, M. A., Langer, W. D., \& Wilson, R. W. 1982, ApJ, 262, 590

Grady, C. A., Schneider, G., Hamaguchi, K., et al. 2007, ApJ, 665, 1391

Hogerheijde, M. R., \& van der Tak, F. F. S. 2000, A\&A, 362, 697

Hollenbach, D., Johnstone, D., Lizano, S., \& Shu, F. 1994, ApJ, 428, 654

Isella, A., Testi, L., Natta, A., et al. 2007, A\&A, 469, 213

Jonkheid, B., Faas, F. G. A., van Zadelhoff, G.-J., \& van Dishoeck, E. F. 2004, A\&A, 428, 511

Kuhn, J. R., Potter, D., \& Parise, B. 2001, ApJ, 553, L189

Lacy, J. H., Knacke, R., Geballe, T. R., \& Tokunaga, A. T. 1994, ApJ, 428, L69

Malfait, K., Bogaert, E., \& Waelkens, C. 1998, A\&A, 331, 211

Mannings, V., \& Sargent, A. I. 1997, ApJ, 490, 792

Meeus, G., Waters, L. B. F. M., Bouwman, J., et al. 2001, A\&A, 365, 476

Ossenkopf, V., \& Henning, T. 1994, A\&A, 291, 943

Piétu, V., Guilloteau, S., \& Dutrey, A. 2005, A\&A, 443, 945

Piétu, V., Dutrey, A., Guilloteau, S., Chapillon, E., \& Pety, J. 2006, A\&A, 460, L43

Piétu, V., Dutrey, A., \& Guilloteau, S. 2007, A\&A, 467, 163

Raman, A., Lisanti, M., Wilner, D. J., Qi, C., \& Hogerheijde, M. 2006, AJ, 131, 2290

Sault, R. J., Teuben, P. J., \& Wright, M. C. H. 1995, in Astronomical Data Analysis Software and Systems IV, ed. R. A. Shaw, H. E. Payne, \& J. J. E. Hayes, ASP Conf. Ser., 77, 433

Simon, M., Dutrey, A., \& Guilloteau, S. 2000, ApJ, 545, 1034

Sylvester, R. J., Skinner, C. J., Barlow, M. J., \& Mannings, V. 1996, MNRAS, 279,915

Thi, W. F., van Dishoeck, E. F., Blake, G. A., et al. 2001, ApJ, 561, 1074

van Zadelhoff, G.-J. 2002, Ph.D. Thesis, Leiden University

van Zadelhoff, G.-J., Aikawa, Y., Hogerheijde, M. R., \& van Dishoeck, E. F. 2003, A\&A, 397, 789

Weingartner, J. C., \& Draine, B. T. 2001, ApJ, 548, 296

Wilson, T. L., \& Rood, R. 1994, ARA\&A, 32, 191 